\title{
Indoor Tracking in WLAN Location with TOA Measurements
}

\author{
Marc Ciurana \\ Universitat Politècnica de Catalunya \\ c/ Jordi Girona 1-3, 08034 \\ Barcelona, Spain \\ +34 934017808 \\ mciurana@entel.upc.edu
}

\author{
Francisco Barceló \\ Universitat Politècnica de Catalunya \\ c/ Jordi Girona 1-3, 08034 \\ Barcelona, Spain \\ +34 934016010 \\ barcelo@entel.upc.edu
}

\author{
Sebastiano Cugno \\ Universitat Politècnica de Catalunya \\ c/ Jordi Girona 1-3, 08034 \\ Barcelona, Spain \\ +34 934017808 \\ scugno@entel.upc.edu
}

\begin{abstract}
Authors presented recently an indoor location technique based on Time Of Arrival (TOA) obtained from Round-Trip-Time (RTT) measurements at data link level and trilateration. This new approach uses the existing IEEE 802.11 WLAN infrastructure with minor changes to provide an accurate estimation of the position of static wireless terminals. This paper presents advances on how to incorporate tracking capabilities to this approach in order to achieve a noticeable enhancement in the positioning accuracy while maintaining the computational cost low, both essential requirements in some critical applications of indoor pedestrian navigation in which people carrying light mobile devices has to be tracked with precision. Taking as a basis the Discrete Kalman Filter, customizations and optimizations have been designed and presented. Results obtained after conducting extensive simulations fed with actual ranging observables demonstrate the validity and suitability of the researched algorithms and its ability to provide very high performance level in terms of accuracy and robustness.
\end{abstract}

\section{Categories and Subject Descriptors}

C.2.1 [Computer-communication Networks]: Network

Architecture and Design - wireless communication; C.4

[Performance of Systems]: Modeling techniques

General Terms: Algorithms, Measurement, Performance, Design, Experimentation.

Keywords: IEEE 802.11, indoor, Kalman, navigation, positioning, time of arrival, TOA, tracking, WLAN.

\section{INTRODUCTION: PREVIOUS RESEARCH AND GOALS}

Some critical applications and services based on indoor localization -such as emergency rescue, fire brigade or incident managementneed an easy-deployable location system able to provide high positioning accuracy (i.e near to $1 \mathrm{~m}$. of error) in medium and deep indoor environments. Since global (i.e.GPS) and wide-area (i.e.

Permission to make digital or hard copies of all or part of this work for personal or classroom use is granted without fee provided that copies are not made or distributed for profit or commercial advantage and that copies bear this notice and the full citation on the first page. To copy otherwise, or republish, to post on servers or to redistribute to lists, requires prior specific permission and/or a fee.

MobiWAC'06, October 2, 2006, Torremolinos, Malaga, Spain.

Copyright 2006 ACM 1-59593-488-X/06/0010...\$5.00. cellular networks) location systems remain inefficient in indoors, alternative positioning technologies are required. The research challenge corresponds to achieve an indoor location system capable to provide accurate tracking using the existing WLAN infrastructure with minor changes (i.e. taking the advantage of the wide deployment of the IEEE 802.11 standard), avoiding the need for synchronization between access points (e.g. as in TDOA based systems) or long system pre-calibrations (e.g. of a fingerprinting database). Following this trend, the authors presented in a previous paper ([1]) an indoor location technique based on Time Of Arrival (TOA) and trilateration. The system was divided into two subsystems: a) Ranging, in charge of obtaining the distances between the Mobile Terminal (MT) and the Access Points (APs) from TOA estimations obtained with Round-Trip-Time (RTT) measuremnts using IEEE 802.11b link layer frames, b) Positioning, a pure trilateration algorithm (Newton) which calculates the MT's position using the distances already estimated and the APs' known position.

The accuracy of the location estimation and the MT trajectory (not smooth) provided the system [1] can be improved by incorporating tracking features. The intention is to apply tracking principles in a similar way as GPS system does: obtaining benefit from past estimated positions and taking as observables (measured data) the distance estimates for finally obtaining as output the location of the MT. It is out of the scope of this work using tracking algorithms to refine TOA or distance estimates in order to mitigate errors due to Non Line Of Sight (NLOS) conditions, as has been presented in [2] and [3]. In fact, this problem is dealt with in the ranging subsystem of our system. In terms of system architecture, the idea is to use the same ranging subsystem and replacing the positioning engine by the new tracking one. Thus, the overall goal is achieving a very accurate indoor TOA-based tracking system for pedestrian navigation which can be deployed over the existing WLAN infrastructure with minor changes.

A decentralized approach is preferred in order to maximize the system scalability and user privacy, therefore some important design constraints have to be taken into account in order to guarantee a feasible implementation in an energy-constrained and processorlimited terminal: keeping the computational cost at a low level and reducing the complexity of the algorithm as much as possible (i.e. not requiring digital environment maps information). According to this, the reported work corresponds to tailoring and improving existing tracking algorithms (explained in Section 3) -specifically based on Kalman filter- and exhaustively evaluating their performance through simulations (explained in Section 4) fed with actual observables obtained with the existing ranging subsystem. 
Research on tracking mobile devices using Kalman algorithms has produced vast literature ([4], [5]) during the last years, but it has been seldom applied to location in indoor environments. In several contributions that propose Kalman filtering for mobile location, the algorithm is applied to smooth the data measurements instead of using it to directly obtain the target location estimate. In [2] and [3] a biased Kalman filter is used to mitigate the effect of NLOS conditions in TOA based location systems. In [6] a received signal strength indicator (RSSI) based location technique is enhanced with Extended Kalman Filter (EKF) based on pre-calibration of measurement vectors and individual position block usage in final estimation of the target's position. A proposal to apply EKF to estimate the patients' location in a hospital is presented in [7]: the ranging measurements obtained from RSSI are the observables of the filter and the patient's location is the filter output; but only simplistic simulation cases are presented and obtained accuracy is not high. In [8] the performances of Kalman and Particle (which also uses the building's map information) filters over WLAN fingerprinting are compared in order to obtain accurate indoor location. In all above-mentioned approaches Kalman filtering seems to be a suitable tool for mobile tracking, but the achieved accuracy is far from $1 \mathrm{~m}$ of error. Our contribution demonstrates that it is possible to achieve very accurate (close to $1 \mathrm{~m}$ error) and robust location estimation with not complex optimizations of this algorithm.

\section{THE TRACKING ALGORITHM}

Despite the existence of more sophisticated filters, Kalman filtering is widely known as being very useful to estimate system states that can only be observed inaccurately: it can be shown that of all possible filters, it is the one that minimizes the variance of the estimation error. Furthermore, the probability distribution of the measurement noise in our ranging system is Gaussian [1], and the premises supposed by the filter (see Section 2.1) are satisfied. Finally, it is not complex to implement it because its recursive nature. For a detailed description of the Kalman filter see [9] and [10].

\subsection{Kalman Filter}

The Kalman filtering bases the state estimation on the weighted average between the measurement $Z_{k}$ at time $t=t_{k}$ and the prediction of the state $\hat{x}_{k}^{-}$from the estimate $\hat{x}_{k-1}$. As we are applying the filter to track the trajectory of a target, the state corresponds to its position and the measurements are the noisy distances estimates between the target and the APs. This weighted average estimation process at a given time works in two steps: a) the filter estimates the current position from past ones (time update or prediction step), b) it obtains the feedback from the noisy measurements in order to improve the accuracy of the estimation (measurement update or correction step).

The equations corresponding to each step are shown in Fig. 1. The weight of every source of information depends on the reliability that can be assumed for each one. The first equation of the prediction step represents the linear process that models the trajectory of the MT; the process noise has covariance matrix $Q$. The matrix $R$ that appears in the first equation (Kalman gain $K_{\mathrm{k}}$ equation) of the correction step corresponds to the measurement noise covariance. Both noises are assumed to be independent of each other, white, and with normal probability distribution. The matrix $A$ of the prediction step corresponds to the transition state matrix. $Q$ and $A$ have to be configured depending on the specific behavior of the target, as below shown. The other matrixes are assumed to change at each step: $H$ is the measurement matrix, $K$ is the Kalman gain matrix, which minimizes the final device position estimation error at each time, and $P$ is the covariance matrix of the position estimation error, which is updated at every step. The algorithm needs an initial estimation of the MT position and the $P$ matrix.

\section{ALGORITHM ADAPTATION}

The Kalman algorithm adaptation consists of defining in detail the prediction step and taking decisions to optimize some specific points of the algorithm design.

Prediction step
$\hat{x}_{k}^{-}=A \cdot \hat{x}_{k-1}$
$P_{k}^{-}=A \cdot P_{k-1} \cdot A^{T}+Q$
$\hat{x}_{k-1}^{\top}, P_{k-1}$ Init.

Figure 1. Scheme of the Discrete Kalman Filter

\subsection{Prediction Step}

$Q$ and $A$ have to be chosen in order that they model as properly as possible the motion behavior of the MT. Theoretically, these matrixes can be defined following three motion models: static, cinematic and random walk. The first one represents processes in which the target is almost static ( $A$ would be the identity matrix and $Q$ null). The second one is suitable for tracking devices with fast speed (represented as $v$ ); $A$ would be null and $Q$ depending on the time between measurements $(T)$ and $v$. The last one can be used if the speed of the target is low. Assuming $v=1 \mathrm{~m} / \mathrm{s}$ (typical for pedestrian motion) it seems clear that the proper model is the last one. In this case, the $A$ matrix is the identity one, and $Q$ depends on $T$ and $v$. Conceptually, this value of $A$ means that the MT does not vary its position from one time to another; while $Q$ is used to model the variation. Hence, $Q$ is a diagonal matrix in which each value different to zero corresponds to the squared maximum variation of a coordinate between two position estimations (this is during $T$ ):

$$
Q=\left(\begin{array}{cc}
(v \cdot T)^{2} & 0 \\
0 & (v \cdot T)^{2}
\end{array}\right) .
$$

\subsection{Improvement of the Prediction Step}

\subsubsection{Motivation}

First evaluations demonstrated that the Kalman algorithm including the prediction step described provided a low enhancement of accuracy with respect to the pure positioning (as shown below in Section 4). For this reason, it was decided to research into improving the method. Since it was difficult to introduce optimizations in the correction step, the work was aimed to improve the way the next position was predicted from the past ones, taking as basis a more geometrical approach.

\subsubsection{Approach}

This new approach relies on supposing that the target is going to follow the straight trajectory defined by the line that joins the last two estimated positions, with the same speed and direction. Hence, 
the next time position $\hat{x}_{k}^{-}$can be predicted applying some basic geometrical laws, as can be appreciated in Fig. 2. Regarding the Kalman equations, the first prediction step is replaced by this new estimation. It must be noticed that the filter order is increased, because $\hat{x}_{k-1}^{-}$and $\hat{x}_{k-2}^{-}$are taken into account. For this reason, when the tracking subsystem starts running using this algorithm the first two position estimations are calculated using Newton, and it is in the third one when the filter really starts working.

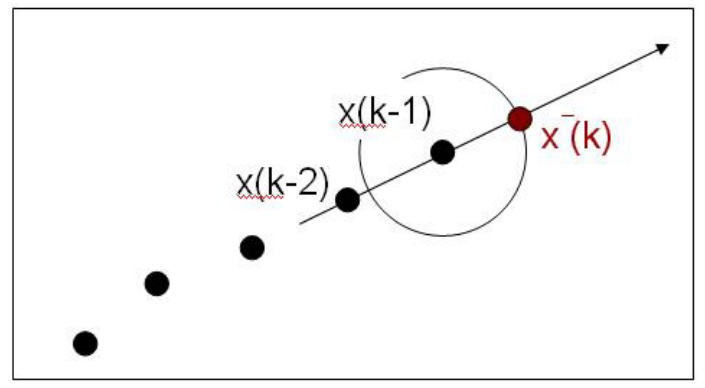

Figure 2. Geometrical scheme of the improved prediction step

An important point of this approach is the estimation of the target speed $v$. In order to guarantee high accuracy, it was decided to estimate $v$ from the last five position estimations, performing an average between the last five $v$ values obtained taking pairs of consecutive positions. With the simulations it was proved that taking more than five past values was not suitable due to the possible actual speed variations, and also that taking less values made decrease the accuracy in most of the cases. So finally the filter takes into account from $\hat{x}_{k-1}^{-}$to $\hat{x}_{k-5}^{-}$.

It has to be noticed that this change on the prediction step involves a redefinition of the prediction error covariance matrix $Q$. In this new situation, the concept that represents this matrix corresponds to the estimation error that can be present if the MT follows the supposed straight trajectory. This error depends on the accuracy of the two last position estimations and the accuracy of the $v$ estimation. For this reason, it is complex to predict the value of this matrix and it has been adjusted empirically through the simulations.

\subsection{Design issues}

For Kalman-based algorithms, an initial MT position estimate is necessary when the filter starts working (filter initialization). Furthermore, it is also necessary at the beginning of each correction step in order to obtain the measurement matrix $H$ and the measurements prefit residuals vector $Z_{\mathrm{k}}$ through a Taylor approximation. Hence, a location method which doesn't require any initial position estimation has to be chosen. The Newton trilateration algorithm has been chosen combined with the Linear Least Squares one, in the same way that it was used for the pure positioning algorithm in [1], because it turned out to provide accurate position estimations without entailing high computational cost.When carrying out the preliminary tests of the algorithms, we realized that it was desirable to perform several iterations in the correction step of the filter in order to achieve a better position estimate; we noticed that five iterations were enough.

\section{PERFORMANCE EVALUATION}

Simulations have been carried out in order to evaluate the performance of the tracking algorithms resulting from the described customizations and improvements running on the system approach proposed in [1]. Furthermore, the Non Linear Least Squares (Newton) trilateration algorithm has also been evaluated in order to evaluate the advantage of tracking results versus pure positioning techniques. The specific implemented algorithms are the following: a) Discrete Kalman filter with the prediction step described in Section 3.1 and the customizations described in Section 3.3 (this algorithm is named Kalman-1), b)Discrete Kalman filter with the improved prediction step described in Section 3.2 and the customizations described in section 3.3 (named Kalman-2) and c)Non Linear Least Squares (Newton)

Two different sets of experiments have been conducted: first, the behavior of algorithms in different types of path intervals and relative situations between the MT and the APs is comparatively analyzed (Section 4.1). Second, reliable results about the tracking algorithms accuracy are obtained (Section 4.2).

The observables that feed the filter (i.e. in the correction step) on every position estimate correspond to the distance estimations from the MT to the three nearest APs, using actual ranging results obtained with the WLAN TOA ranging prototype described in [1] either in LOS and NLOS conditions. Since the distance estimation results could be modeled by a Gaussian distribution with $\mu=$ actual_dist +1.12 , and $\sigma=0.84$ the matrix $R$ of the Kalman filter is:

$$
R=\left(\begin{array}{ccc}
0.84^{2} & 0 & 0 \\
0 & 0.84^{2} & 0 \\
0 & 0 & 0.84^{2}
\end{array}\right) .
$$

In practice, every observable is obtained by calculating a single random value from a normal random variable with the mentioned parameters. Hence, it is essential to perform a large number of runs of a specific route simulation in order to guarantee that the actual ranging model is really used. This way the results of these simulations are fair and theoretically almost the same as the ones obtained with a tracking prototype in an actual indoor environment.

\subsection{Tracking examples}

The main objective of this set of simulations is the performance assessment of the algorithms regarding different types of route intervals (changes of direction, straight trajectories...) and the dependence with the geometry of the MT with respect to the APs (i.e. the GDOP parameter). The best case for tracking algorithms is supposed to be a straight trajectory with APs placed in such a way that a good GDOP is available. However, in actual indoor situations worst cases are likely to occur often. For this purpose, a route is generated (see Fig. 3) in a scenario composed by a squared area of $50 \times 50$ meters with an AP in every corner. The MT speed is variable with average $1 \mathrm{~m} / \mathrm{s}$ (pedestrian) and the positioning step $T$ is set to 1 second. Several changes of direction and bad GDOP zones are included; specifically a part -close to the center of the squared area- in which the GDOP is expected to be so bad due to the collinear situation between the MT and two of the three APs involved in the position calculation. 


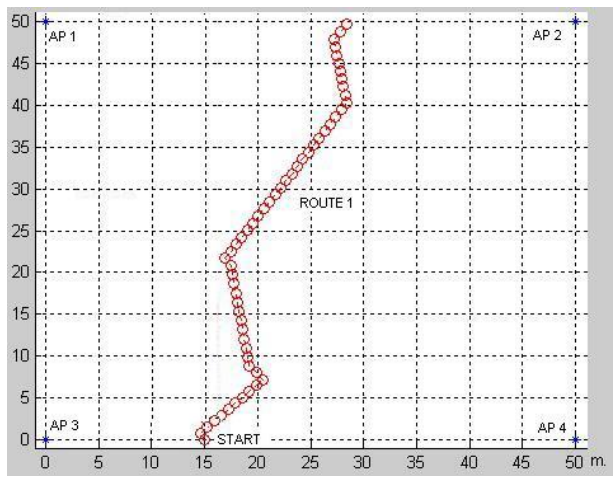

Figure 3. Route for the first type of simulations

\subsubsection{Results}

Fig. 4 shows the average and variance of the position estimate error (in meters), and GDOP for each point of the routes; the points in which a change of direction occurs are highlighted. Since the initialization period of the algorithms has not been taken into account (to avoid edge effects) for this performance assessment, the first position represented corresponds to the sixth position of the route.

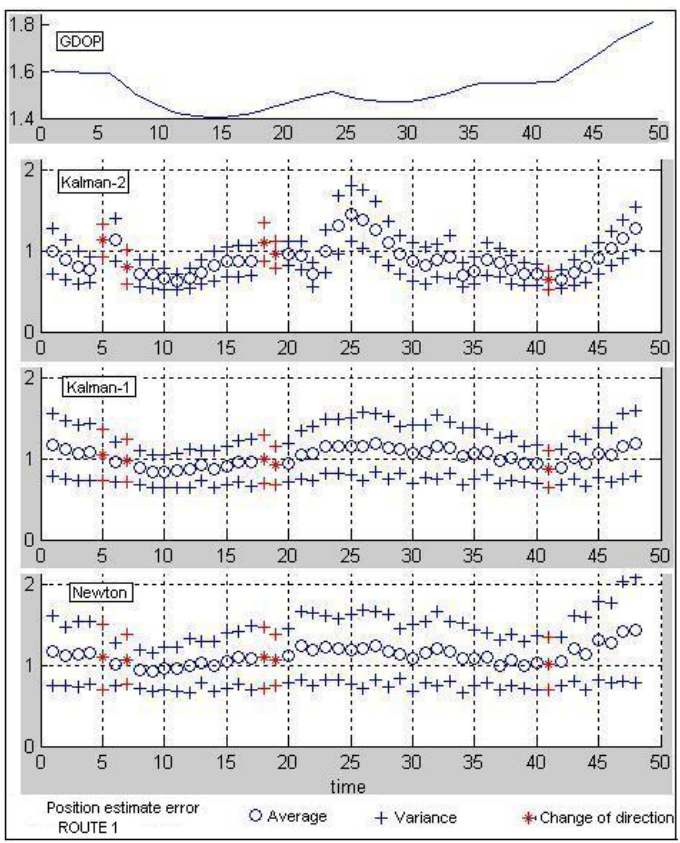

Figure 4. Average and variance of the position estimate error, and GDOP for the generated route

In terms of accuracy, Kalman-2 algorithm outperforms the other ones because the average position estimate error remains lower than 1 meter in most of the points for both routes, while Kalman- 1 and specially the Newton trilateration algorithm normally provide figures very close or higher than 1 meter. As expected, the pure positioning algorithm seems to provide the worst performance.

Analyzing the error figures in the periods immediately subsequent to changes of direction in the actual routes, it is possible to appreciate that in some of them Kalman-1 responds better than Kalman-2 (Newton is out of scope of this analysis, due to its pure positioning nature that makes it insensitive to direction or speed changes): after the 5th and 19th positions of the route, Kalman-2 provides its less accurate position estimates while Kalman-1 does not suffer this effect. The explanation for this can be found taking a look in the GDOP graph: the combination of noticeable change of direction and bad GDOP negatively impacts in the provided accuracy of Kalman-2, while Kalman-1 is able to almost maintain its usual accuracy. In situations of good GDOP, both algorithms respond well to changes of direction.

If we focus on the performance dependence on the GDOP when the actual trajectory describes an almost straight line, it can be seen than the performance degradation due to bad GDOP is a little bit higher in Kalman-2 than in Kalman-1 (see the last positions of the route in Fig. 4), but even in this case the average error figures provided by Kalman- 2 are not worse than the ones provided by Kalman- 1 .

The expected behavior was that the impact of any relevant change of direction using Kalman-2 would be higher than for Kalman-1 due to its severe position prediction step, which is forcing in major degree to finally estimate a position that follows the straight line drawn by past target trajectory. However, it can be stated that Kalman-1 outperforms Kalman-2 only in points with bad GDOP in which a noticeable change of direction occurs. As expected, Kalman-2 provides very accurate position estimates in smoothed trajectories (around $0.7 \mathrm{~m}$. of average error). Furthermore, the variance of the position estimate error obtained with Kalman-2 is always the lowest.

Finally Fig. 5 shows an interval of the actual MT trajectory and the estimated ones obtained with Newton and Kalman-2 algorithms. It can be easily appreciated that the later provides an erratic path whereas the former is able to achieve a smoothed trajectory very similar to the actual one.

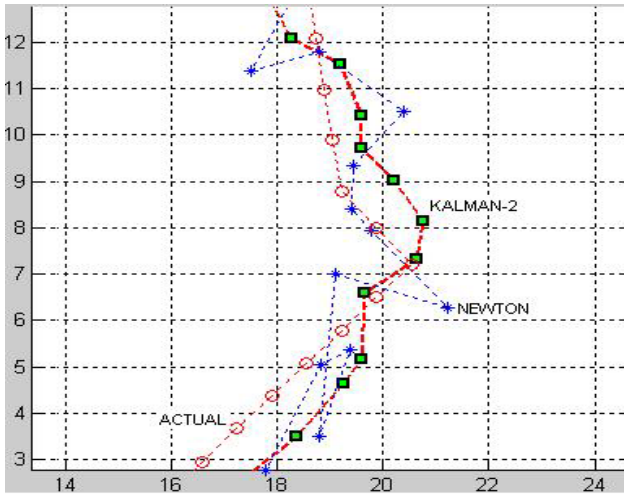

Figure 5. Actual and estimated trajectories

\subsection{Performance assessment}

Through this part of simulations it is aimed to obtain reliable and representative positioning accuracy figures for both Kalman-based tracking algorithms and the pure positioning one, in order to assess the performance enhancement that specially is expected to provide the improved prediction step approach (Kalman-2). Taking into account the results obtained in the first phase of simulations, a large number of routes (5000) with bad GDOP zones and probable changes of direction were generated following a motion model as similar as possible to a real behavior of a pedestrian. The motion algorithm is managed by the following rules: a) probability of changing the direction at a given time is governed by a geometrical random distribution. with probability of change $=0.1, \mathrm{~b}) \cdot$ speed of the MT is a normal random variable of mean $1 \mathrm{~m} / \mathrm{s}$ and variance 0.2 
$\mathrm{m} / \mathrm{s}$, c) change of direction can be up to 30 degrees respect the followed straight line. The scenario is composed by a squared area of $50 \times 50 \mathrm{~m}^{2}$ with an AP in every corner. The positioning step $T$ is set to 1 second.

\subsubsection{Results}

Fig. 6 shows the Cumulative Distribution Function (CDF) of the absolute positioning error for the algorithms. It can be seen that the Discrete Kalman algorithm with the improved prediction step provides the best accuracy, specifically less than $0.9 \mathrm{~m}$. of absolute positioning error for the $66 \%$ of the cases (one sigma), and less than $1.4 \mathrm{~m}$. for the $90 \%$. Comparing with Newton, the improvement seems to be noticeable, because it provides $1.8 \mathrm{~m}$. and $1.2 \mathrm{~m}$. for the $90 \%$ and the $66 \%$ respectively.

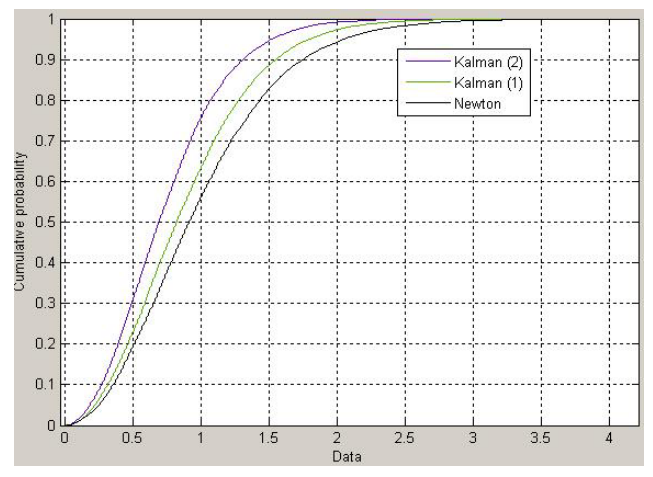

Figure 6. CDF of the absolute positioning error

\section{CONCLUSIONS}

The use of a non complex tracking algorithm in an indoor WLAN location system based on TOA has been treated in this paper. The main objective of incorporating tracking to the TOA location technique presented in [1] is to improve the achieved positioning accuracy. The Discrete Kalman Filter has been taken as a basis for obtaining a proper algorithm that provides good performance given our system constraints. Accordingly, two different adaptations and optimizations of this filter have been evaluated through simulations (comparing them with Newton algorithm) fed with actual TOAbased ranging observables, in terms of positioning accuracy and robustness to changes of direction and GDOP. Results show that both tracking algorithms outperform Newton, specially the Kalman algorithm with an improved prediction step, which is able to enhance the accuracy half a meter. This achieved accuracy (less than $0.9 \mathrm{~m}$ of error for the $66 \%$ of cases) really encourages adopting this improved Kalman Filtering as the tracking algorithm in our indoor WLAN. The global result consists of an indoor TOA-based tracking system for pedestrian navigation which can be deployed over the existing WLAN infrastructure with minor changes, providing high robustness and accuracy. Further research is necessary to deal with important issues of the system performance such as the lack of localization availability in situations of less than 3 APs being in the range of the MT.

\section{ACKNOWLEDGMENTS}

This research has been funded by the Spanish Government through the MECD and FEDER project TIC 2003-01748, and by the EC under the Sixth FP IST LIAISON Integrated Project. The authors thank Pere Ramos-Bosch and Dagoberto Salazar of the group of Astronomy and Geomatics (gAGE) at UPC for their help and advising.

\section{REFERENCES}

[1] F.Izquierdo, M.Ciurana, F.Barceló, J.Paradells, E.Zola "Performance evaluation of a TOA-based trilateration method to locate terminals in WLAN". Proc. IEEE ISWPC 2006, pp. 217-222.

[2] B.Long Le, K.Ahmed, H.Tsuji "Mobile Location Estimator with NLOS Mitigation Using Kalman Filtering" Mar 17-19 2003, IEEE WCNC New Orleans, , USA, 2003

[3] N.J. Thomas, D.G.M. Cruickshank, D.I. Laurenson, “A robust Location Estimator Architecture with Biased Kalman Filtering of TOA Data for Wireless Systems" IEEE 6th Int. Symp. on Spread-Spectrum Tech \& Appli NJIT, pp. 296-300, New Jersey, USA, Sept 6-8, 2000

[4] M. Najar, J. Vidal, A. Kjellstrom. "Kalman Tracking for UMTS Mobile Location". Proc. IST Summit 2001, p. 230-235.

[5] P.Pathirana, A.Savkin and S.Jha "Mobility modelling and trajectory prediction of cellular networks with mobile base stations". Proc. of the ACM International Symposium on Mobile Ad Hoc Networking and Computing (Mobihoc 2003), Annapolis, Maryland, USA, pp. 213-221.

[6] Q. Cai, A. Mitiche, and J. K. Aggarwal, "Tracking human motion in an indoor environment" in Proceedings of the 2nd International Conference on Image Processing (ICIP'95), pp. $215\{218,1995$.

[7] J. Latvala, J. Syrjärinne, S. Niemi, J. Niittylahti , "Patient Tracking in a Hospital Environment Using Wireless Stations and Extended Kalman Filtering", Proceedings of the 1999 Middle East Conference on Networking.

[8] F.Evennou and F. Marx "Improving Positioning capabilities for indoor environments with WiFi”, IST Summit 2005.

[9] I. Guvenc, C. T. Abdallah, R. Jordan, and O. Dedeoglu, "Enhancements to RSS based indoor tracking systems using kalman filters," in Proc. of International Signal Processing Conference and Global Signal Processing Expo, 2003.

[10] R. Kalman, "A new approach to linear filtering and prediction problems”, Trans. ASME, J. Basic Eng. 82D, pp. 35--45, 1960. 